14,19

\title{
Фазовые переходы в молекулярных кристаллах парафинового ряда: генэйкозане и докозане
}

\author{
(C) B.М. Егоров, В.А. Марихин \\ Физико-технический институт им. А.Ф. Иофрфе РАН, \\ Санкт-Петербург, Россия \\ E-mail: victor_egorov1@inbox.ru
}

(Поступила в Редакцию 9 июня 2016 г.)

Проведен сравнительный анализ фазовых переходов в нормальных парафинах - генэйкозане $\left(\mathrm{C}_{21} \mathrm{H}_{44}\right)$ и докозане $\left(\mathrm{C}_{22} \mathrm{H}_{46}\right)$ - методом дифференциальной сканирующей калориметрии. Устранение методических погрешностей позволило получить истинные значения термодинамических параметров фазовых переходов и выявить их природу. Проведен количественный анализ профилей скачков теплоемкости на основе теории размытых фазовых переходов первого рода и теории Ландау для переходов второго рода.

Работа выполнена при финансовой поддержке РФФИ (код проекта 16-03-00493).

Выяснение природы и эволюции фазовых переходов (ФП) в твердых телах, включая полимеры и длинноцепочечные молекулярные кристаллы, составляет одно из важнейших направлений физики твердого тела. Однако значительная дисперсия характеристик полимеров как на молекулярном уровне, так и на различных иерархических уровнях надмолекулярной организации приводит к заметному размытию параметров, характеризующих ФП. Это существенно затрудняет выявление природы ФП и установление закономерностей фазовых превращений в зависимости от структуры полимеров и, как следствие, приводит к невозможности анализа экспериментальных данных на основе имеющихся в настоящее время теорий фазовых переходов первого и второго рода.

Изучение ФП в молекулярных кристаллах цепочечного строения открывает новые возможности для установления обобщающих количественных закономерностей взаимосвязи структура-термодинамические свойства, поскольку в настоящее время исследования могут быть проведены на монодисперсных гомологических рядах молекулярных кристаллов различной химической природы с высокой степенью очистки. Значительный интерес представляют гомологические ряды алифатических углеводородов и их производных, включая нормальные н-парафины $\left(\mathrm{C}_{n} \mathrm{H}_{2 n+2}\right)$.

Одним из эффективных методов исследования ФП является метод дифференциальной сканирующей калориметрии (ДСК), широко используемый для изучения молекулярных кристаллов различного типа, в том числе парафинов [1-5]. Вместе с тем в большинстве опубликованных работ не были устранены методические погрешности, связанные с эффектом термосопротивления при ДСК-измерениях, который может приводить к существенным искажениям значений термодинамических характеристик изучаемых молекулярных кристаллов. Кроме того, как правило, не приводились и не анализировались ДСК-исследования в циклах нагревание-охлаждение, которые, как показано в настоящей работе, позволяют выявить природу фазовых переходов, а также получить истинные профили эндо- и экзопиков на термограммах. Последнее обстоятельство является очень важным для анализа экспериментальных данных на основе развитых в настоящее время теорий размытых фазовых переходов первого рода [6,7] и фазовых переходов второго рода [8].

Образцы генэйкозана и докозана приобретены на фирме „Sigma“. Тепловые свойства исследовались на калориметре DSC-2 „Perkin-Elmer“ в атмосфере азота при варьировании скоростей нагревания и охлаждения в диапазоне $5-0.3 \mathrm{~K} / \mathrm{min}$. Температурная шкала калибровалась по точкам плавления льда $(273.1 \mathrm{~K})$ и индия $(429.7 \mathrm{~K})$, шкала теплового потока - по теплоемкости лейкосапфира. Измерения проводились в области температур $250-400 \mathrm{~K}$. Небольшая масса образцов для испытаний, составлявшая 1-3 mg, обеспечивала минимальное термическое сопротивление калориметрической ячейки и уменьшала методические погрешности.

На рис. 1 представлены кривые ДСК, полученные при нагревании и охлаждении образцов генэйкозана и докозана. Как видно из рисунка, при нагревании переход из кристаллического состояния в расплав для обоих парафинов осуществляется в два этапа: на кривых ДСК виден дублет с температурами максимумов $T_{\max 1}$ и $T_{\max 2 .}$ Обратный процесс изменения фазового состояния расплав-кристалл при охлаждении происходит также в два этапа (температуры минимумов соответствующих им экзотермических пиков $T_{\min 1}$ и $\left.T_{\min 2}\right)$. Следует отметить, что форма эндотермического пика перехода из кристаллического состояния в расплав для каждого из исследованных материалов имеет свои особенности.

Такая картина в общем виде соответствует результатам опубликованных работ, поэтому можно ожидать, что эндотермический пик с $T_{\max 1}$ при нагревании связан, по-видимому, с фазовым переходом в твердом состоянии $T_{\mathrm{s}-\mathrm{s}}$, за которым следует при температуре $T_{\max 2}$ плавление образца. Соответственно при охлаждении из 


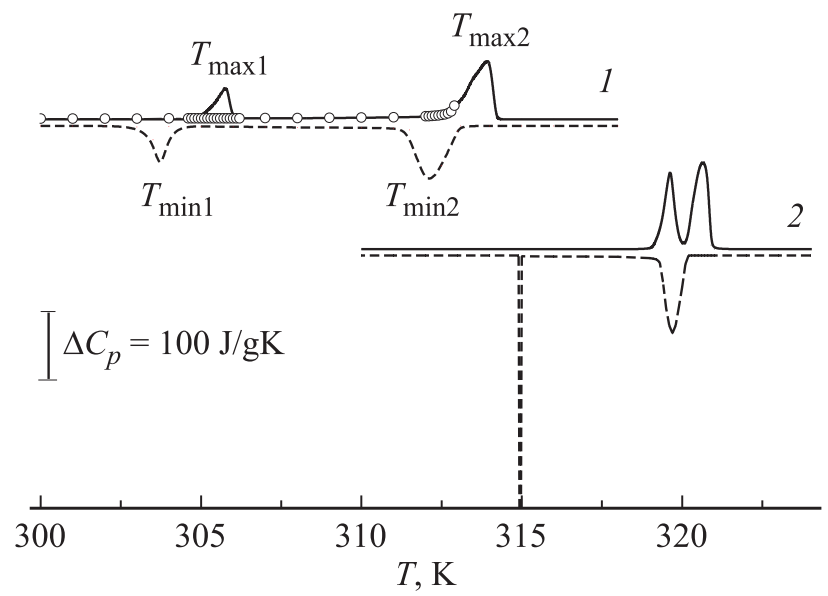

Рис. 1. Кривые ДСК, полученные при нагревании (сплошные линии) и охлаждении (штриховые линии) образцов генэйкозана (1) и докозана (2). Точки - рассчитанная по формуле (1) зависимость, пояснения в тексте. Скорость сканирования $V=1.25 \mathrm{~K} / \mathrm{min}$.

расплава в этом случае наблюдается обратная картина: при $T_{\min 2}$ происходит их кристаллизация и при $T_{\min 1}$ реализуется твердофазный переход.

Для понимания физической природы регистрируемых на термограммах переходов необходимо прояснить ситуацию с наблюдаемым температурным несовпадением пиков: на кривых ДСК видны смещения относительных положений эндо- и экзопиков в циклах нагревание-охлаждение $\left(T_{\max 1}-T_{\min 1}\right.$ и $\left.T_{\max 2}-T_{\min 2}\right)$. Оказалось, что смещения (так называемый эффект гистерезиса) имеют для каждого из пиков дублета свою природу, обусловленную как методическими, так и физическими причинами. Известно [9], что при использовании метода ДСК возникают методические погрешности (смещение пиков), связанные с наличием термосопротивления испытываемого образца в калориметрической ячейке, которое существенно зависит от массы образца и скорости сканирования. В работе [10] показано, что эти методические погрешности могут быть устранены следующим образом: по экспериментальным данным при вариации скоростей сканирования $V$ (нагревания или охлаждения) строятся зависимости $T_{\max , \min }=f\left(V^{1 / 2}\right)$, которые при отсутствии структурных трансформаций должны быть линейными. Экстраполяция линейных зависимостей к $V \rightarrow 0$ позволяет получить истинные, методически неискаженные значения температур переходов.

На рис. 2 представлены подобные зависимости для исследованных образцов генэйкозана $(a)$ и докозана (b) в циклах нагревание-охлаждение. Полученные по этим зависимостям экстраполяционные (истинные) значения температур фазовых переходов оказались равны для генэйкозана $T_{\max 1}^{r}=305.1 \mathrm{~K}, T_{\max 2}^{r}=$ $=313.1 \mathrm{~K}, T_{\min 1}^{r}=304.6 \mathrm{~K}, T_{\min 2}^{r}=313.1 \mathrm{~K}$, а для докозана $T_{\max 1}^{r}=319.4 \mathrm{~K}, T_{\max 2}^{r}=320.1 \mathrm{~K}, T_{\min 1}^{r}=317.0 \mathrm{~K}$, $T_{\min 2}^{r}=320.1 \mathrm{~K}$. На основании этих данных можно за- ключить, что в действительности для вторых экстремумов для образцов генэйкозана и докозана температурный гистерезис в циклах нагревание-охлаждение отсутствует, а наблюдаемая на экспериментальных кривых ДСК разница в их положении на температурной шкале обусловлена методическими причинами. В то же время устранение методической погрешности с помощью экстраполяционных зависимостей позволило выявить для низкотемпературного перехода в этих образцах наличие гистерезиса.

Энтальпия $\Delta H$ и энтропия $\Delta S$ переходов в методе ДСК не зависят от скорости сканирования по температуре и определяются соотношениями $\Delta H_{\exp }=\int C_{p}(T) d T$ и $\Delta S_{\exp }=\int C_{p}(T) d(\ln T)$. Суммарные значения энтальпии $\Delta H_{\text {sum }}$ и энтропии $\Delta S_{\text {sum }}$ переходов для генэйкозана оказались равны $242 \mathrm{~J} / \mathrm{g}$ и $0.781 \mathrm{~J} / \mathrm{g} \cdot \mathrm{K}$, для докозана $234 \mathrm{~J} / \mathrm{g}$ и $0.794 \mathrm{~J} / \mathrm{g} \cdot \mathrm{K}$.

Как известно [8], наличие или отсутствие температурного гистерезиса какого-либо физического свойства, в том числе для пиков на температурной зависимости теплоемкости, является одним из признаков, по которому фазовые переходы относят соответственно к переходам первого или второго рода. Поэтому отсутствие гистерезиса для вторых экстремумов прямо указывает на принадлежность высокотемпературных пиков в дублетах к переходам второго рода. Если не использовать экстраполяционные зависимости $T_{\max , \min }=f\left(V^{1 / 2}\right)$, применяемые в настоящей работе, а пользоваться лишь данными, полученными при одной скорости сканирования по температуре (рис. 1), то высокотемпературные пики можно ошибочно также отнести к переходам первого рода. C другой стороны, наличие истинного гистерезиса для низкотемпературных пиков позволяет на основании данных ДСК отнести этот переход к твердофазному (структурному) превращению первого рода, связанному с изменением типа симметрии кристалла. Ранее [2,11-13] методом рентгеновской дифракции было показано, что в температурном интервале, соответствующем пику теплоемкости с $T_{\max 1}$, действительно происходит изменение симметрии молекулярных кристаллов: орторомбическая элементарная субъячейка превращается в гексагональную в кристаллических сердечниках ламелей.

Как видно из рис. 1 , экзопики $C_{p}$ для генэйкозана и докозана, соответствующие фазовым переходам первого и второго рода, в цикле охлаждения (переход из расплава в кристаллическое состояние) четко разнесены на температурной шкале. С другой стороны, в цикле нагревания (переход из кристаллического состояния в расплав) соответствующие эндопики могут быть либо четко разделены (генэйкозан), либо частично сливаться (докозан), что в последнем случае существенно ограничивает возможности их корректной обработки. Если переходы первого и второго рода разнесены на температурной шкале, то в этом случае пик твердофазного перехода можно анализировать независимо от перехода второго рода. Нами разработан методический прием, позволяющий проводить экспериментальное разделение 


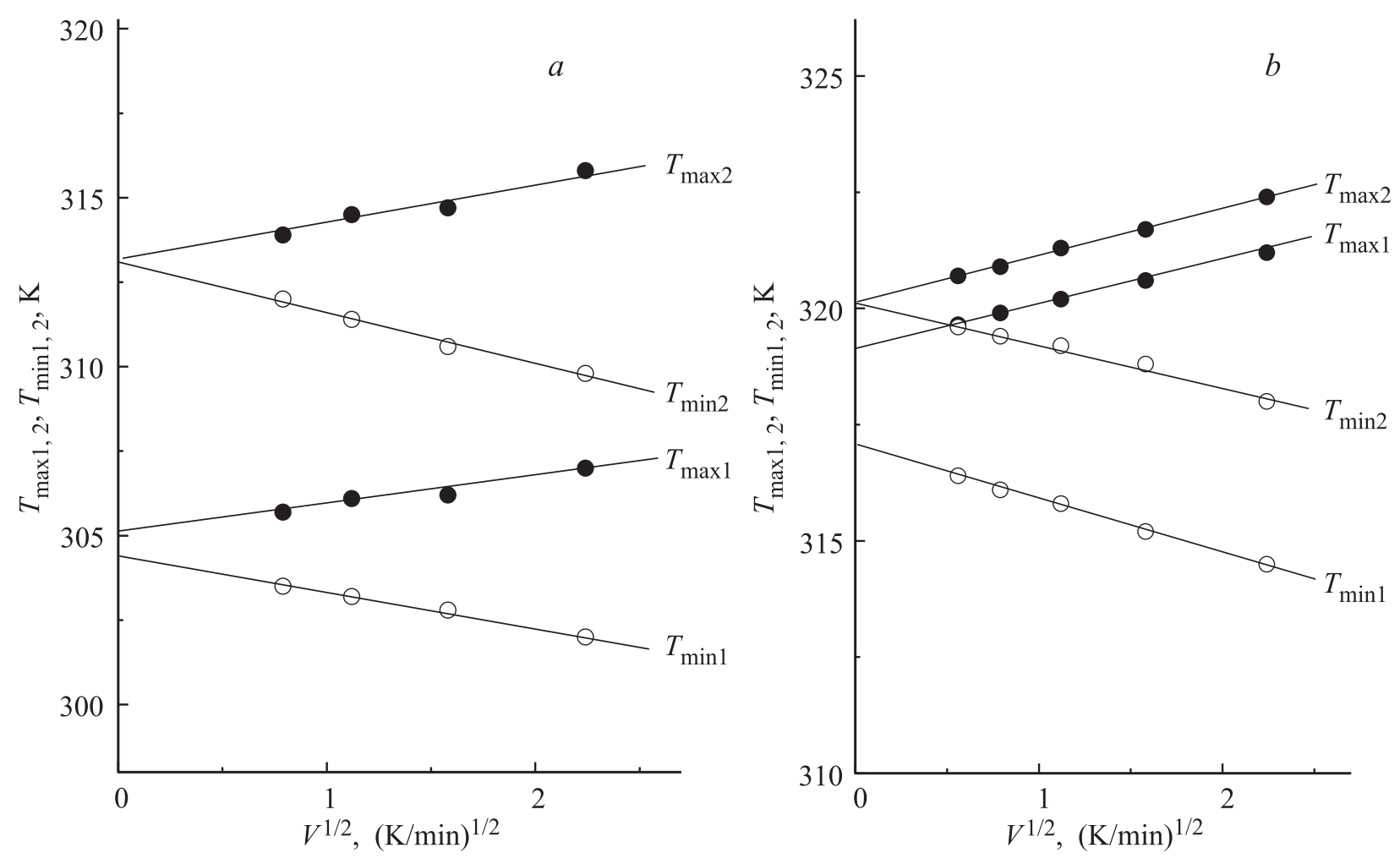

Рис. 2. Зависимости температур фазовых переходов от скорости сканирования для генэйкозана $(a)$ и докозана $(b)$. Темные точки - нагревание, светлые - охлаждение.

накладывающихся пиков. Для этого мы воспользовались свойством переходов первого рода - наличием температурного гистерезиса, т.е. разницы между истинными температурами переходов при нагревании и охлаждении $\Delta T=T_{\max }^{r}-T_{\min }^{r}$.

Для экспериментального разделения пиков для докозана была проведена процедура, показанная на рис. 3: образец нагревался до состояния расплава при $T=323 \mathrm{~K}$ (кривая 1), после чего охлаждался, минуя экзотермический пик при $T_{\min 2}$, до температуры $316 \mathrm{~K}$ (кривая 3). Таким образом, фиксировалось промежуточное фазовое состояние, при котором в цикле охлаждения твердофазный переход первого рода еще не произошел, а упорядочение, связанное с переходом второго рода, уже осуществилось. Повторный нагрев от температуры $316 \mathrm{~K}$ до температуры расплава (кривая 2) в этом случае показывает „чистый“ переход второго рода. По разнице между пиками на первичных и повторных кривых нагревания можно выделить эндотермические эффекты, которые отвечают лишь фазовым переходам первого рода.

Как было показано в работах Ландау [8] для фазовых переходов второго рода, пик $C_{p}$ должен иметь $\lambda$-образную форму (напоминающую греческую букву $\lambda$ ). Подобная форма пика наряду с отсутствием описанного выше эффекта гистерезиса является специфическим признаком переходов второго рода. Зависимость теплоемкости $C_{p}(T)$ при температурах $T<T_{\max }$ должна в этом случае описываться степенной функцией типа

$$
C_{p}(T)=D\left(T_{0}-T\right)^{-\alpha},
$$

где $D, T_{0}$ и $\alpha-$ константы, $T_{0}-$ имеет смысл истинной температуры фазового перехода второго рода.

Наиболее простым способом проверки соответствия экспериментальных данных соотношению (1) является построение графика в двойных логарифмических координатах $\ln \left(C_{p} D^{-1}\right)-\ln \left(T_{0}-T\right)$; в этом случае должна получиться прямолинейная зависимость, по наклону которой определяется показатель $\alpha$ степенной зависимости. Как видно из рис. 4, наши экспериментальные данные для генэйкозана действительно хорошо укладываются на прямую с наклоном $\alpha=0.5$. Кроме того, на рис. 1 (кривая 1 ) для низкотемпературной части пика $T_{\max 2}$ точками представлена рассчитанная по соотношению (1) зависимость $C_{p}$, которая наилучшим образом совпадает с экспериментальными данными при значениях параметров $D=9.5, \alpha=0.5$ и $T_{0}=313.0 \mathrm{~K}$. Поскольку расчетная степенная зависимость может быть продолжена и в область температур, соответствующих фазовому переходу первого рода, это позволяет после вычитания из экспериментальной зависимости $C_{p}$ расчетной составляющей в области $T_{\max 1}$ уточнить форму пика, соответствующего фазовому переходу первого рода.

Таким образом были выделены эндотермические пики, отвечающие структурным фазовым переходам первого 


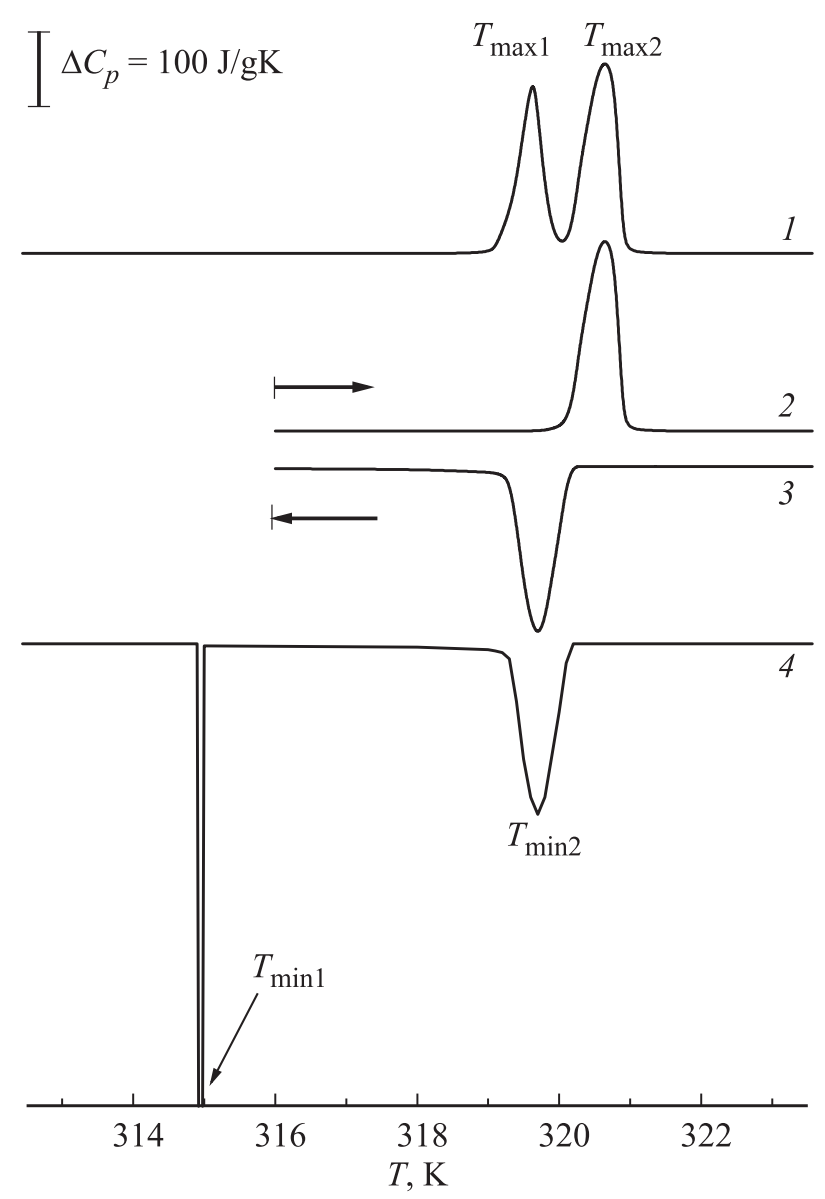

Рис. 3. Кривые ДСК образцов докозана, полученные при нагревании $(1,2)$ и охлаждении $(3,4)$.

рода (рис. 5). Для докозана пик оказался симметричным, по форме напоминающим так называемый $\Lambda$-образный пик (рис. $5, b)$. Однако для генэйкозана (рис. $5, a$ ) форма пика оказалась несимметричной, что позволяет предположить наличие по крайней мере двух составляющих. Эти особенности формы пиков рассмотрены далее.

Анализ пиков, полученных указанным выше способом, проводился согласно термодинамической теории самосогласованного поля $[6,7,14]$ применительно к $\Lambda$-образным размытым переходам первого рода. Тот факт, что переход становится размытым, означает, что изменение фазового состояния происходит не внезапно, а постепенно и осуществляется в пределах определенного температурного интервала. Теория анализирует изменение в фазовом состоянии системы взаимодействующих частиц различных фаз, в частности образование и последующий рост устойчивых зародышей новой фазы в матрице старой фазы. Механизм нуклеации гетерогенный, причем зародыши новой фазы возникают на различного типа дефектах в кристаллах, что приводит к появлению межфазной границы, которая является характерным признаком перехода первого рода. Основная идея теории состоит в том, что в объеме старой фазы происходит ло- кализация многочисленных флуктуаций в ограниченном объеме в виде стабильных зародышей новой фазы - так называемых элементарных объемов перехода $\omega$. В дальнейшем по мере развития перехода движение межфазной границы осуществляется путем последовательных добавлений зародышей с объемом $\omega$ на возникшую межфазную границу. Образование зародышей-доменов требует изменения температуры на малую величину, которая определяется энергией, необходимой для возникновения таких областей. Это и приводит к размытию перехода по температуре. Например, для сегнетоэлектрических материалов было показано, что элементарные объемы превращения сопоставимы с объемом так называемой области Кенцига $\left(\sim 10^{-18}-10^{-17} \mathrm{~cm}^{3}\right)$ и по своему масштабу находятся на мезоскопическом уровне $[15,16]$.

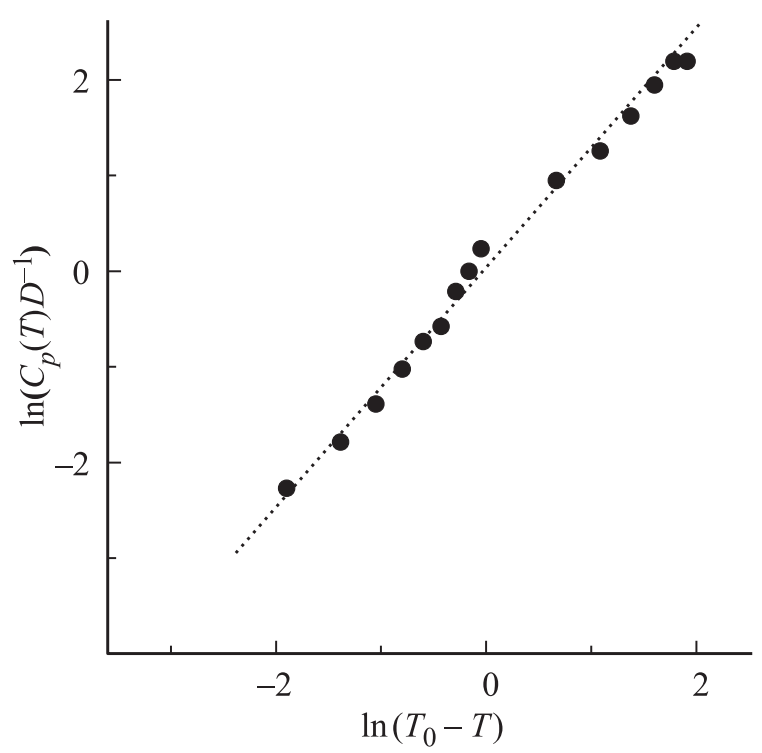

Рис. 4. Зависимость $C_{p}(T)$ для генэйкозана в двойных логарифмических координатах.

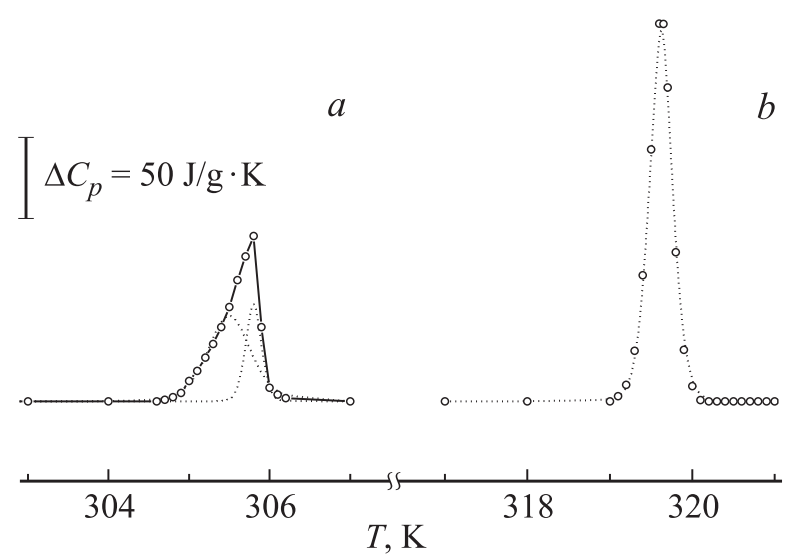

Рис. 5. Эндотермические пики, отвечающие твердофазным переходам в генэйкозане $(a)$ и докозане $(b)$. Точки - экспериментальные данные, линии - результат расчета по зависимости (2). 
Оказалось, что размеры устойчивых зародышей $\omega$ можно определить по форме пиков $C_{p}$, соответствующих переходам первого рода. В работе [17] получено соотношение для температурной зависимости теплоемкости при размытом фазовом переходе в виде

$$
\begin{aligned}
\Delta C_{p}(T)= & 4 \Delta C_{m} \exp \left[B\left(T-T_{o}\right) / T_{o}\right] \\
& \times\left\{1+\exp \left[B\left(T-T_{o}\right) / T_{o}\right]\right\}^{-2},
\end{aligned}
$$

где $T_{o}$ - температура фазового перехода первого рода, $\Delta C_{m}-$ максимальное значение теплоемкости при $T=T_{o}, B-$ атермический параметр.

Расчет теплоемкости по соотношению (2) проводился для докозана по пику со значениями $T_{o}=319.5 \mathrm{~K}$ и $\Delta C_{\max }=240 \mathrm{~J} / \mathrm{g} \cdot \mathrm{K}$, соответствующими температуре и амплитуде максимума пика. В несимметричном по форме пике для генэйкозана производилось разделение на два пика симметричной $\Lambda$-образной формы при условии равенства энтальпии экспериментально полученного пика сумме энтальпий двух симметричных пиков. Варьированием параметров $T_{o}, \Delta C_{\max }$ и $B$ для каждого из симметричных пиков можно было получить совпадение либо с правым (высокотемпературным), либо с левым (низкотемпературным) плечом пика. Оказалось, что наилучшее согласие экспериментальной зависимости $\Delta C_{p}$ с суммой симметричных пиков получалось при совпадении левого (низкотемпературного) плеча пика $\Delta C_{p} \mathrm{c}$ низкотемпературным плечом одного из симметричных пиков. Эти особенности пиков $\Delta C_{p}$ рассмотрены далее.

На рис. 5 показаны результаты расчета $\Lambda$-образных пиков по соотношению (2) и экспериментальные зависимости теплоемкости $\Delta C_{p}(T)$. Наилучшее совпадение расчетных и экспериментальных зависимостей наблюдалось при следующих значениях параметров: для докозана $B=3600$; для генэйкозана для каждого из двух пиков соответственно $B_{1}=1750, T_{o 1}=305.5 \mathrm{~K}$, $\Delta C_{m 1}=55 \mathrm{~J} / \mathrm{g} \cdot \mathrm{K}$ и $B_{2}=6000, T_{o 2}=305.8 \mathrm{~K}, \Delta C_{m 2}=$ $=75 \mathrm{~J} / \mathrm{g} \cdot \mathrm{K}$.

Параметр $B$ в приведенном выше соотношении (2) содержит наиболее интересную информацию о физической природе фазового перехода, поскольку он связан с величиной пика теплоемкости $\Delta C_{m}$

$$
\Delta C_{m}=q_{o} B / 4 T_{o},
$$

(где $q_{o}$ - теплота превращения [17]) и элементарным объемом превращения $\omega$

$$
B=\omega \rho q_{o} / k T_{o},
$$

где $k-$ постоянная Больцмана, $\rho-$ плотность. Параметр $B$ оказывается структурно-чувствительным, поскольку определяет в материалах с размытыми фазовыми переходами объемы зародышей новой фазы [7]. Из соотношения (3) можно найти значения удельной теплоты (энтальпии) превращения, из соотношения (4) - элементарный объем превращения $\omega$ (полагая в первом приближении, что плотность кристаллов парафинов $\left.\rho \sim 0.8 \mathrm{~g} \cdot \mathrm{cm}^{-3}[1]\right)$. Результаты расчета по этим соотношениям следующие: для докозана $q_{o}=86 \mathrm{~J} / \mathrm{g}$, $\omega \cong 230 \mathrm{~nm}^{3}$; для генэйкозана $q_{o 1}=39 \mathrm{~J} / \mathrm{g}, \omega_{1} \cong 240 \mathrm{~nm}^{3}$ и $q_{o 2}=15 \mathrm{~J} / \mathrm{g}, \omega_{2} \cong 2000 \mathrm{~nm}^{3}$.

Оценка числа молекул, участвующих в образовании зародыша новой фазы в докозане и на первой стадии фазового перехода в генэйкозане, показывает, что в элементарных объемах превращения $\omega \sim 230-240 \mathrm{~nm}^{3}$ в области перехода первого рода содержится $\sim 450-500$ молекул, т.е. область превращения захватывает весьма большой домен. Еще большее число молекул $(\sim 4000)$ участвует в образовании зародышей новой фазы на второй стадии фазового перехода в генэйкозане.

Рассмотрим возможные локальные места возникновения устойчивых зародышей новой фазы. Форма и местоположение домена или зародыша новой фазы определяются соотношением объемной и поверхностной энергий надмолекулярной структуры, состоящей из стопок ламелей, т. е. кристаллических сердечников, и межламелярных ,прослоек“. Сердцевину ламелей образуют плотноупакованные „стержни“ - длинноцепочечные молекулы, которые связаны между собой так называемым универсальным (ван-дер-ваальсовым) взаимодействием между метиленовыми группами. Межслоевые прослойки для парафинов состоят из концевых групп - $\mathrm{CH}_{3}$. Из соображений минимизации внутренней энергии естественно предположить, во-первых, что зародыш новой фазы может располагаться в пределах одной ламели. Во-вторых, фазовая граница не может проходить через часть молекулы, т. е. через наиболее сильные химические связи в метиленовой части молекул $(\mathrm{C}-\mathrm{C}$ и $\mathrm{C}-\mathrm{H})$, и, по-видимому, проходит через относительно слабые ван-дер-ваальсовы связи между метиленовыми группами соседних молекул и концевыми группами $\mathrm{CH}_{3}$. Таким образом, домен можно представить в виде параллелепипеда, состоящего из параллельно упакованных „стержней“, в основании которых содержится 400-500 метиленовых цепей.

Наличие второй стадии фазового перехода в генэйкозане с $\omega_{2} \cong 2000 \mathrm{~nm}^{3}$ можно объяснить следующим образом. На первой стадии происходит „высыпание“ значительного количества доменов новой фазы объемом $\sim 240 \mathrm{~nm}^{3}$ и реализуется фазовое превращение в большей части кристалла. Это следует из соотношения теплот превращения на первой и второй стадиях $q_{o 1} / q_{o 2} \sim 2.5$. Накопление большого количества доменов новой фазы объемом $\sim 240 \mathrm{~nm}^{3}$ приводит к усилению эффекта их действия, т.е. „кумулятивному эффекту“. В основе этого эффекта лежит изменение соотношения объемной и поверхностной энергии в оставшейся, т. е. не претерпевшей фазового превращения части кристалла. Для сохранения баланса между объемной и поверхностной энергиями, по-видимому, должна повыситься доля более высокой торцевой поверхностной энергии, т.е. площадь торцевой поверхности ламели, и соответственно объем домена. 


\section{Список литературы}

[1] D.M. Small. The physical chemistry of lipids. Plenum Press, N. Y.-London (1986). 262 p.

[2] T. Yamamoto, K. Nozaki, T. Hara. J. Phys. Chem. 92, 631 (1990).

[3] Y. Ogawa, N. Nakamura. Bull. Chem. Soc. Jpn. 72, 943 (1999).

[4] В.М. Егоров, В.А. Марихин, Л.П. Мясникова. Высокомолекуляр. соединения Б 47, 2191 (2005).

[5] В.М. Егоров, В.А. Марихин, Л.П. Мясникова. Высокомолекуляр. соединения А 48, 2138 (2006).

[6] Б.Н. Ролов, В.Э. Юркевич. Физика размытых фазовых переходов. Изд-во Ростов. ун-та, Ростов н/Д (1983). 317 с.

[7] Г.А. Малыгин. УФН 171, 187 (2001).

[8] Л.Д. Ландау, Е.М. Лифшиц. Статистическая физика. Наука, M. (1976). 514 c.

[9] V.A. Bershtein, V.M. Egorov. Differential scanning calorimetry of polymers: physics, chemistry, analysis, technology. Ellis Horwood, N. Y. (1994). 253 p.

[10] K. Illers. Eur. Polym. J. 10, 911 (1974).

[11] S. Abrahamsson, G. Larsson, E. Sydov. Acta Cryst. 13, 770 (1960).

[12] R. Popovitz-Biro, J.L. Wang, J. Majewski, E. Shavit, L. Leiserowitz, M. Lahav. J. Am. Chem. Soc. 116, 1179 (1994).

[13] J-L. Wang, F. Leveiller, D. Jacqueman, K. Kjaer, J. Als-Nielsen, M. Lahav, L. Leiserowitz. J. Am. Chem. Soc. 116, 1192 (1994).

[14] М. Фишер. Природа критического состояния. Мир, М. (1968). $221 \mathrm{c}$.

[15] В. Кенциг. Сегнетоэлектрики и антисегнетоэлектрики. ИЛ, М. (1960). 347 с.

[16] В.Я. Фрицберг. Изв. АН СССР. Сер. физ. 47, 698 (1983).

[17] Г.А. Малыгин. ФТТ 43, 1911 (2001). 